\title{
Etude comparée de la sensibilité au sel d'un triticale et d'une orge
}

Bruno TOURAINE (l) \& Meimoun AMMAR

I.N.A.T., Laboratoire d'Agronomie, 43, av. Charles Nicolle, Tunis, Tunisie.

\begin{abstract}
Nous avons cultivé jusqu'au stade floraison une variété de triticale sélectionnée en Tunisie («110») et, pour comparaison, l'orge " Martin », très répandue dans ce pays, dans des pots irrigués avec différentes doses de $\mathrm{NaCl}$. L'orge s'est confirmée assez tolérante, mais le triticale est apparu plus résistant. Ce dernier n'accumule pas $\mathrm{Na}^{+}$dans ses organes aériens, au contraire de l'orge, réagissant ainsi comme une plante " exclusive " caractère pourtant généralement associé à la sensibilité à $\mathrm{NaCl}$. La plus grande sélectivité du triticale en faveur $\mathrm{de}^{+}$se manifeste plus particulièrement dans les organes jeunes, dernières feuilles et, surtout, épis. Ces résultats suggèrent que la différence de sensibilité à $\mathrm{NaCl}$ des deux plantes s'explique par leur comportement vis-àvis des transports d'ions, mais dans un sens tout à fait imprévu. Le triticale devrait donc constituer un matériel intéressant pour les physiologistes étudiant les problèmes de résistance au sel. D'un point de vue agronomique, nous pensons que sa grande tolérance à la salinité devrait encourager sa culture dans des zones semi-arides comme la Tunisie.
\end{abstract}

Mots clés additionnels : Tolérance à $\mathrm{NaCl}$, transpiration, plante exclusive, plante inclusive, sélectivité $\mathrm{K} / \mathrm{Na}$.
Comparison of salt tolerance in triticale and barley.

A Tunisian cultivar of triticale ( 110 ") and, for comparison, barley cv. " Martin ", widespread in Tunisia, were grown till flowering in pots watered with different $\mathrm{NaCl}$ concentrations. Barley was confirmed as fairly $\mathrm{NaCl}$-tolerant, but triticale appeared more resistant. It did not accumulate $\mathrm{Na}^{+}$in its shoots, unlike barley, and thus reacted as an excluder, behaviour usually associated with $\mathrm{NaCl}$ sensitivity. The higher selectivity of triticale for $\mathrm{K}^{+}$was shown particularly by young organs, flag leaves and ears. These results suggest that the diflerence in $\mathrm{NaCl}$ sensitivity between the two plants could be explained by their ion-transport behaviour, but in a completely unexpected way. Triticale appeared as a particularly interesting plant for physiologists working on the problems of salt resistance. From the point of view of agriculture, we think that its great tolerance should encourage its cultivation in semi-arid countries such as Tunisia.

Additional key words : $\mathrm{NaCl}$ tolerance, transpiration, excluder, includer, $\mathrm{K} / \mathrm{Na}$ selectivity.

\section{INTRODUCTION}

Depuis plusieurs années, la ferme expérimentale de Frétissa constate que, dans le Nord de la Tunisie, le triticale pourrait avantageusement remplacer l'orge. L'intérêt agronomique du triticale est souligné par une étude comparative récente des rendements et des qualités nutritionnelles des principales céréales cultivées en France (VERMOREL \& BERNARD, 1979).

La plus grande partie du territoire tunisien est caractérisée par un climat semi-aride à aride. Dans ces conditions, la teneur de la solution du sol en sels - et par-

(1) Adresse actuelle : I.N.R.A., Laboratoire de Biochimie et de Physiologie Végétales, F 34060 Montpellier Cedex. ticulièrement en $\mathrm{NaCl}$ - est souvent anormalement élevée. Mais il n'existe à notre connaissance aucune donnée sur la sensibilité du triticale au sel. Nous avons voulu étudier le comportement de cette céréale dans un milieu chargé en $\mathrm{NaCl}$, en le comparant à celui de l'orge, considérée comme l'une des glycophytes les plus tolérantes (MAAS \& HOFFMAN, 1977).

Dans un environnement salé, les plantes doivent faire face à un dilemme : pour remédier aux effets de l'abaissement du potentiel osmotique du milieu, le végétal doit accumuler des électrolytes et cette accumulation pose des problèmes de toxicité dont la résolution dépend de l'efficacité des mécanismes régulateurs (GREENWAY \& MunNS, 1980). A côté des halophytes - plantes pouvant se développer en présence de très 
fortes concentrations de sels de sodium -, les auteurs distinguent 2 groupes de végétaux : les glycophytes, appelées " exclusives ", qui ne peuvent pas accumuler $\mathrm{Na}^{+}$dans leurs feuilles, et celles, dites " inclusives " qui, comme l'orge, peuvent l'utiliser pour rétablir leur équilibre hydrique. Ce comportement permet aux " inclusives " de supporter de plus fortes doses de sel (MAAS \& NiEmAN, 1978). Mais ces plantes sont alors confrontées à des déséquilibres nutritionnels, principalement $\mathrm{K}^{+} / \mathrm{Na}^{+}$et $\mathrm{NO}_{3}{ }^{-} / \mathrm{Cl}^{-}$ (STOREY \& WYN JONES, 1978). Ainsi les glycophytes les plus tolérantes seront celles qui, tout en utilisant $\mathrm{Na}^{+}$comme osmoticum, garderont une forte sélectivité en faveur de $\mathrm{K}^{+}$(WYN JONES \& STOREY, 1978). La stratégie utilisée par les végétaux pour éviter les problèmes d'excès d'ions tout en réalisant leur équilibre osmotique est la compartimentation cellulaire : $\mathrm{Na}^{+}$est préférentiellement accumulé dans la vacuole (JESCHKE, 1979). Chez les glycophytes tolérantes, on discerne également une compartimentation à l'échelle de la plante : la teneur en $\mathrm{Na}^{+}$dans les organes jeunes et actifs reste faible (WINTER \& LAUCHLI, 1982).

Dans cette étude, nous avons cherché à mettre en évidence les déséquilibres hydriques et nutritionnels qui affectent le triticale et l'orge en milieu chargé en $\mathrm{NaCl}$ et qui pourraient expliquer des différences de sensibilité à la salinité.

\section{MATÉRIEL ET MÉTHODES}

Les plantules de triticale « 110 » et d'orge «Martin ", obtenues après germination en boîtes de Petri, sont repiquées fin novembre par 4 dans des pots de $5 \mathrm{~kg}$ contenant un mélange $1: 1 \mathrm{de}$ sable et de sol de l'I.N.A.T. (argileux). Les pots sont placés sous serre. Ils sont irrigués à leur capacité de rétention $(1,1 \mathrm{l})$ avec de l'eau distillée. A partir de la $2^{\mathrm{e}}$ semaine, les arrosages hebdomadaires ( $1 \mathrm{l}$ par pot) contiennent $\mathrm{NaCl}$ à différentes concentrations et, une fois sur 4 , la solution nutritive suivante: $\mathrm{MgSO}_{4} 1 \mathrm{mM}, \mathrm{Ca}\left(\mathrm{NO}_{3}\right)_{2} 2 \mathrm{mM}$, $\mathrm{KNO}_{3} 2 \mathrm{mM}, \mathrm{FeSO}_{4} 6 \mu \mathrm{M}, \mathrm{H}_{3} \mathrm{BO}_{3} 5 \mu \mathrm{M}, \mathrm{MnSO}_{4}$ $1 \mu \mathrm{M}, \mathrm{ZnSO}_{4} 0,5 \mu \mathrm{M}, \mathrm{CuSO}_{4} 0,5 \mu \mathrm{M}$ et molybdate d'ammonium $10 \mathrm{nM} ; \mathrm{K}_{2} \mathrm{HPO}_{4}$ est mélangé au sol avant remplissage des pots, à raison de $100 \mathrm{ppm}$ (environ 80 unités $\mathrm{P}_{2} \mathrm{O}_{5} / \mathrm{ha}$ ). Les doses de $\mathrm{NaCl}$ utilisées sont : $0,1,5,3$ et $4,5 \mathrm{~g} \cdot 1^{-1}(0,25,50$ et $75 \mathrm{mM})$. Nous disposons pour chaque traitement et pour chaque variété de 4 pots. A partir de la $11^{\mathrm{e}}$ semaine, 2 pots de chaque série reçoivent $\mathrm{NaCl}$ à $12 \mathrm{~g} .1^{-1}(200 \mathrm{mM})$, alors que les 2 autres continuent d'être irrigués avec la dose initiale. Dans la suite, les traitements seront désignés par « $O$ », « $1,5 », ~ « 3 », ~ « 4,5 », ~ « 0-12 »$, « 1,5-12», « 3-12», « 4,5-12».

La transpiration est estimée par la perte d'eau des pots dont la surface est couverte d'un film de plastique noir. Les parties aériennes sont récoltées à la floraison, le 16 mars pour le triticale et du 10 au 20 avril pour l’orge. Elles sont divisées en 4 lots : épis, dernières feuilles, autres feuilles et tiges. Après détermination du poids de matière sèche (g MS), nous avons analysé l'azote total par la méthode de Kjeldahl sur $0,5 \mathrm{~g}$ MS broyée et dosé les ions $\mathrm{Na}^{+}, \mathrm{K}^{+}$et $\mathrm{Ca}^{+}+$extraits par $\mathrm{HNO}_{3} 0,1 \mathrm{~N}$ avec un photomètre à émission de flamme
Eppendorf. Les résultats présentés sont les moyennes des 8 répétitions dont nous disposons pour chaque organe et shaque traitement.

\section{RÉSULTATS}

\section{A. Production}

Le triticale « 110 » est plus précoce que l'orge "Martin ", ce qui est en accord avec les observations en plein champ : sur le traitement " $O$ », ces plantes épient respectivement le 25 février et le 20 mars. Les traitements salés n'ont qu'un effet limité sur la précocité de ces 2 céréales: la date d'épiaison de l'orge est légèrement retardée (de $9 \mathrm{j}$ pour le traitement « 4,5-12 ») et celle du triticale n'est pas modifiée. Alors que chez le triticale la croissance en longueur du maître-brin n'est pratiquement pas affectée par la présence de $\mathrm{NaCl}$, chez l'orge elle est inhibée de 25 p. 100 par le traitement « 4,5-12 ». De même, l'élévation de la concentration de $\mathrm{NaCl}$ dans l'eau d'irrigation se traduit par une baisse de production de matière sèche dans les organes aériens chez l'orge, mais non chez le triticale (fig. 1).

\section{B. Teneur en eau et transpiration}

Contrairement à ce que STOREY \& WYN JONES (1978) ont observé sur plantules d'orge, la teneur en eau des organes aériens de nos plantes n'est pas sensiblement affectée par les fortes doses de sel : elle reste comprise entre 62 et $64 \mathrm{p} .100 \mathrm{chez}$ le triticale et entre 64 et 66 p. 100 chez l'orge.

Chez les 2 céréales, la transpiration diminue avec l'augmentation de la salinité (fig. 2). Mais cet effet n'est marqué que pour la dose extrême de $\mathrm{NaCl}$,
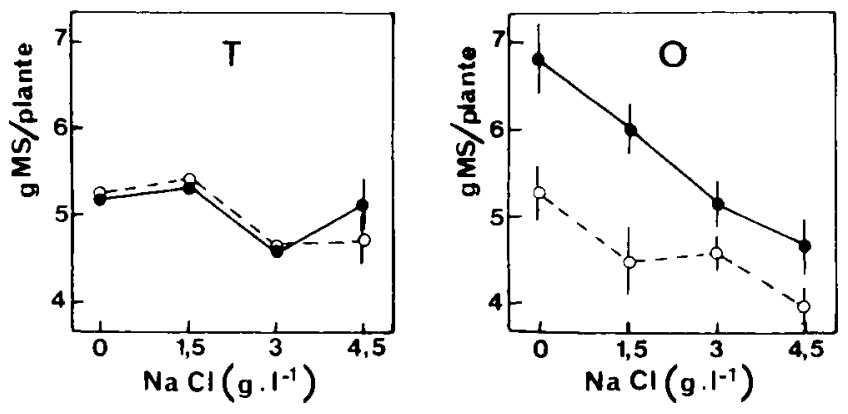

Figure 1

Production de matière sèche dans les parties aériennes de triticale " $110 »(T)$ et d'orge « Martin » $(O)$ récoltés au stade floraison (respectivement le 16 mars et du 10 au 20 avril), en fonction de la teneur en $\mathrm{NaCl}$ de la solution d'irrigation. Les plantes reçoivent la dose initiale de $\mathrm{NaCl}$ jusqu'à la récolte (@) ou $12{\mathrm{~g} . \mathrm{l}^{-1}}^{-1}$ à partir du 16 février (0). Les barres verticales représentent les intervalles de confiance calculés pour $p=0,05$.

Dry weight production in shoots of triticale "110" (T) and barley "Martin" $(O)$ harvested at flowering (respectively March 16th and April 10th to 20th), in relation to $\mathrm{NaCl}$ content of irrigation solution. The plants receive $\mathrm{NaCl}$ at the initial concentration until harvest (full symbols), or at 12 g.t-1 after February 16th (open symbols). Confidence limits calculated for $p=0.05$. 

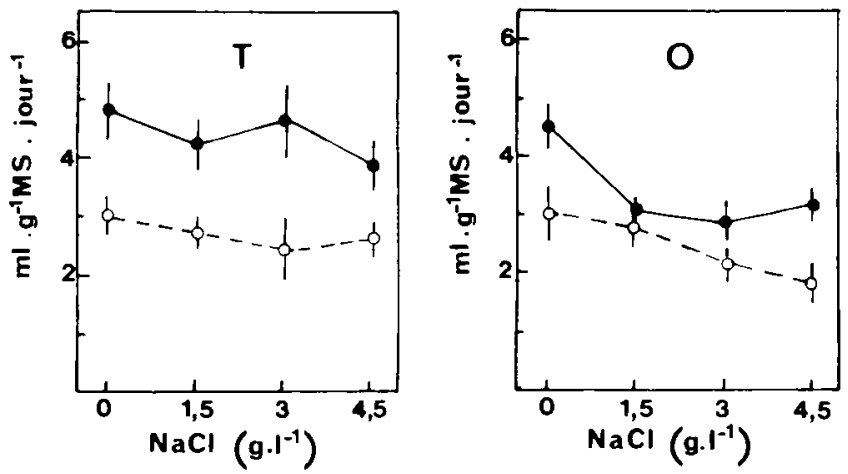

Figure 2

Effet des traitements salés sur la transpiration de plantes de triticale " $110 »(T)$ et d'orge «Martin» (O) au stade épiaison. La signification des symboles et des barres verticales est donnée dans la légende de la figure 1 .

Effect of salt treatments on transpiration of triticale " 110 " $(T)$ and barley "Martin" $(O)$ at heading. Symbols are defined in the caption of figure 1. Confidence limits calculated for $p=0.05$.

12 g. $1^{-1}$, et plus particulièrement chez le triticale (fig. 2 : comparer la courbe en traits pleins et celle en tirets pour chaque plante).

\section{Composition cationique}

Le tableau 1 montre que l'orge se comporte comme une "inclusive " et le triticale comme une " exclusive ». Parallèlement à l'augmentation de la teneur en sodium des tissus de l'orge, nous observons une baisse de la teneur en potassium ; au contraire, le triticale accumule des quantités croissantes de cet élément lorsque la dose extérieure de $\mathrm{NaCl}$ s'élève (tabl. 1). Ces tendances générales sont plus ou moins marquées selon l'organe considéré : le tableau 2 montre que les épis de l'orge peuvent accumuler des quantités importantes de sodium, alors que cet ion reste pratiquement exclu des épis du triticale.
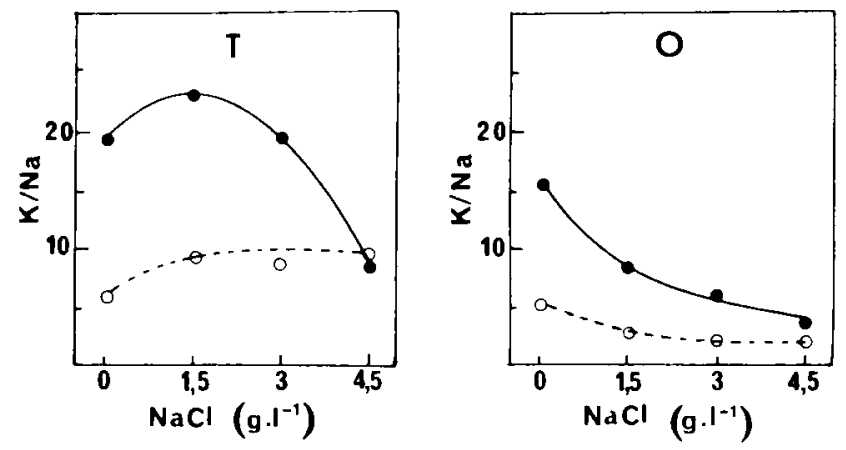

Figure 3

Effet des traitements salés sur le rapport $K / \mathrm{Na}$ (en $\mathrm{mol} / \mathrm{mol}$ ) dans les parties aériennes de triticale " $l 10$ " $(T)$ et d'orge "Martin» (O). La signification des symboles et des barres verticales est donnée dans la légende de la figure 1.

Effect of salt treatments on the $\mathrm{K} / \mathrm{Na}$ ratio $(\mathrm{mol} / \mathrm{mol}$ ) in the shoots of triticale " 110 " $(T)$ and barley "Martin" $(O)$. Symbols are defined in the caption of figure 1. Confidence limits calculated for $p=0.05$.

La très forte sélectivité du triticale en faveur de $\mathrm{K}^{+}$ est mise en évidence par l'évolution du rapport $\mathrm{K} / \mathrm{Na}$ chez les 2 espèces étudiées (fig. 3). Des analyses de variance montrent que la concentration initiale de $\mathrm{NaCl}$ a un effet hautement significatif sur ce rapport ; pour une même dose, l'élévation finale de la concentration de sel à $12 \mathrm{~g} .1^{-1}$ a aussi un effet hautement significatif, sauf lorsque l'on compare les traitements « 4,5 » et « 4,5-12» chez le triticale.

\section{Azote}

Les traitements salés ne semblent affecter ni la teneur moyenne en azote des parties aériennes (tabl. 3) ni la distribution de cet élément entre les différents organes (résultats non présentés).

\section{TABLEAU 1}

Teneurs moyennes en cations ( $\mu$ mol.g ${ }^{-1}$ MS) dans les parties aériennes de triticale " 110 " et d'orge "Martin " récoltées au stade floraison (respectivement le 16 mars el du 10 au 20 avril). Les plantes sont irriguées avec une solution contenant $0,1,5,3$ ou $4,5 \mathrm{g.t} \mathrm{l}^{-1}$ de $\mathrm{NaCl}$ jusqu'au 16 février ; au-delà de cette date, soit la dose initiale de NaCl est apportée jusqu'à la récolte (traitements « $0 », 《 1,5 》, 《 3 », 44,5 »)$, soit l'irrigation se poursuit avec 12 g. $1^{-1}$ de $\mathrm{NaCl}$ (traitements «0-12», «1,5-12», «3-12», « 4,5-12»). Les intervalles de confiance sont calculés pour $p=0,05$.

Average cation content (umol.g-1 DW) of shoots of triticale "110" and barley "Martin" harvested at flowering (respectively on March 16th

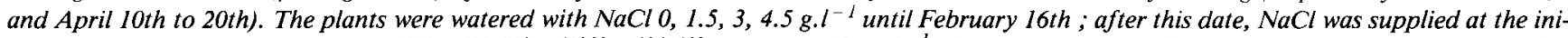
tial concentration (treatments " 0 ", "1.5", "3", "4.5"), or at 12 g.l-1 (treatments "0-12", "1.5-12", "3-12", "4.5-12"), Confidence limits calculated for $p=0.05$.

\begin{tabular}{|c|c|c|c|c|c|c|}
\hline \multirow[b]{2}{*}{ Traitement } & \multicolumn{3}{|c|}{ Triticale } & \multicolumn{3}{|c|}{ Orge } \\
\hline & $\mathrm{K}$ & $\mathrm{Na}$ & $\mathrm{Ca}$ & $\mathrm{K}$ & $\mathrm{Na}$ & $\mathrm{Ca}$ \\
\hline 0 & $389+21$ & $20 \pm 5$ & $32 \pm 5$ & $405 \pm 20$ & $26 \pm 5$ & $37 \pm 5$ \\
\hline 1,5 & $372 \pm 29$ & $16 \pm 2$ & $29 \pm 5$ & $402 \pm 15$ & $50 \pm 7$ & $35 \pm 4$ \\
\hline 3 & $394 \pm 30$ & $20 \pm 4$ & $32 \pm 5$ & $395 \pm 15$ & $62 \pm 4$ & $28 \pm 3$ \\
\hline 4,5 & $417 \pm 44$ & $49 \pm 8$ & $37 \pm 3$ & $346 \pm 28$ & $105 \pm 19$ & $22 \pm 4$ \\
\hline $\begin{array}{ll}0 & -12\end{array}$ & $422 \pm 34$ & $73 \pm 23$ & $35 \pm 3$ & $423 \pm 16$ & $80 \pm 13$ & $36 \pm 3$ \\
\hline $1,5-12$ & $424 \pm 30$ & $44 \pm 5$ & $49 \pm 4$ & $418 \pm 19$ & $135 \pm 13$ & $39 \pm 4$ \\
\hline $\begin{array}{ll}3 & -12\end{array}$ & $428 \pm 21$ & $49 \pm 7$ & $32 \pm 4$ & $403 \pm 8$ & $165 \pm 10$ & $28 \pm 6$ \\
\hline $4,5-12$ & $422 \pm 40$ & $45 \pm 6$ & $44 \pm 6$ & $370 \pm 14$ & $154 \pm 14$ & $37 \pm 5$ \\
\hline
\end{tabular}


TABLEAU 2

Teneurs en cations ( $\mu$ mol.g $\left.{ }^{-I} M S\right)$ dans les épis $(E)$, dernières feuilles $\left(F_{l}\right)$, autres feuilles $(F)$ et tiges $(T)$ de triticale « $110 »$ et d'orge « Martin » récoltés au stade floraison.

Les traitements sont définis dans la légende du tableau 1 . Les intervalles de confiance sont calculés pour $p=0,05$.

Average cation content ( $\mu$ mol.g $\mathrm{g}^{-I} \mathrm{DW}$ ) of ears $(E)$, flag leaves $\left(F_{1}\right)$, other leaves $(F)$ and stems $(T)$ of triticale "110" and barley "Martin" harvested at flowering.

Treatments defined as in table 1. Confidence limits calculated for $p=0.05$.

\begin{tabular}{|c|c|c|c|c|c|}
\hline \multirow[b]{2}{*}{ Organe } & \multirow[b]{2}{*}{ Traitement } & \multicolumn{2}{|c|}{ Triticale } & \multicolumn{2}{|c|}{ Orge } \\
\hline & & K & $\mathrm{Na}$ & $\mathrm{K}$ & $\mathrm{Na}$ \\
\hline \multirow{8}{*}{$\mathrm{E}$} & 0 & $191 \pm 12$ & $6 \pm 1$ & $187 \pm 12$ & $7 \pm 1$ \\
\hline & 1,5 & $220 \pm 21$ & $5 \pm 1$ & $188 \pm 10$ & $22 \pm 2$ \\
\hline & 3 & $198 \pm 10$ & $9 \pm 2$ & $169 \pm 11$ & $29 \pm 1$ \\
\hline & 4,5 & $271 \pm 25$ & $14 \pm 4$ & $145 \pm 10$ & $38 \pm 3$ \\
\hline & $\begin{array}{ll}0 & -12\end{array}$ & $288 \pm 17$ & $6 \pm 2$ & $230 \pm 13$ & $15 \pm 2$ \\
\hline & $1,5-12$ & $304 \pm 20$ & $4 \pm 1$ & $213 \pm 16$ & $26 \pm 3$ \\
\hline & $\begin{array}{ll}3 & -12\end{array}$ & $292 \pm 12$ & $8 \pm 3$ & $193 \pm 7$ & $37 \pm 3$ \\
\hline & $4,5-12$ & $302 \pm 36$ & $5 \pm 1$ & $158 \pm 10$ & $52 \pm 4$ \\
\hline \multirow{8}{*}{$F_{1}$} & 0 & $316 \pm 15$ & $19 \pm 3$ & $543 \pm 20$ & $44 \pm 6$ \\
\hline & 1,5 & $295 \pm 17$ & $23 \pm 1$ & $528 \pm 17$ & $49 \pm 5$ \\
\hline & 3 & $358 \pm 16$ & $27 \pm 5$ & $536 \pm 19$ & $56 \pm 6$ \\
\hline & 4,5 & $263 \pm 24$ & $31 \pm 6$ & $511 \pm 30$ & $75 \pm 8$ \\
\hline & $\begin{array}{ll}0 & -12\end{array}$ & $371 \pm 23$ & $44 \pm 17$ & $577 \pm 14$ & $70 \pm 7$ \\
\hline & $1,5-12$ & $385 \pm 18$ & $39 \pm 2$ & $571 \pm 18$ & $91 \pm 9$ \\
\hline & $\begin{array}{ll}3 & -12\end{array}$ & $392 \pm 10$ & $42 \pm 7$ & $565 \pm 9$ & $128 \pm 13$ \\
\hline & $4,5-12$ & $301 \pm 27$ & $43 \pm 8$ & $524 \pm 12$ & $132 \pm 14$ \\
\hline \multirow{8}{*}{$\mathrm{F}$} & 0 & $462 \pm 24$ & $21 \pm 7$ & $612 \pm 24$ & $48 \pm 9$ \\
\hline & 1,5 & $440 \pm 15$ & $29 \pm 4$ & $573 \pm 21$ & $67 \pm 8$ \\
\hline & 3 & $444 \pm 30$ & $58 \pm 7$ & $588 \pm 16$ & $79 \pm 3$ \\
\hline & 4,5 & $420 \pm 40$ & $67 \pm 9$ & $555 \pm 32$ & $128 \pm 13$ \\
\hline & $\begin{array}{ll}0 & -12\end{array}$ & $390 \pm 35$ & $40 \pm 11$ & $652 \pm 17$ & $69 \pm 8$ \\
\hline & $1,5-12$ & $402 \pm 22$ & $52 \pm 7$ & $637 \pm 22$ & $130 \pm 9$ \\
\hline & $\begin{array}{ll}3 & -12\end{array}$ & $414 \pm 20$ & $53 \pm 5$ & $615 \pm 10$ & $143 \pm 8$ \\
\hline & $4,5-12$ & $402 \pm 32$ & $48 \pm 6$ & $586 \pm 16$ & $124 \pm 8$ \\
\hline \multirow{8}{*}{$\mathrm{T}$} & 0 & $414 \pm 20$ & $22 \pm 6$ & $379 \pm 14$ & $26 \pm 2$ \\
\hline & 1,5 & $396 \pm 34$ & $14 \pm 2$ & $382 \pm 14$ & $54 \pm 5$ \\
\hline & 3 & $379 \pm 33$ & $16 \pm 3$ & $367 \pm 13$ & $60 \pm 4$ \\
\hline & 4,5 & $465 \pm 48$ & $45 \pm 8$ & $320 \pm 17$ & $112 \pm 22$ \\
\hline & $\begin{array}{ll}0 & -12\end{array}$ & $481 \pm 39$ & $108 \pm 30$ & $420 \pm 16$ & $105 \pm 18$ \\
\hline & $1,5-12$ & $473 \pm 40$ & $53 \pm 7$ & $409 \pm 17$ & $142 \pm 17$ \\
\hline & $\begin{array}{ll}3 & -12\end{array}$ & $484 \pm 42$ & $54 \pm 9$ & $381 \pm 6$ & $187 \pm 12$ \\
\hline & $4,5-12$ & $457 \pm 45$ & $52 \pm 7$ & $359 \pm 15$ & $171 \pm 15$ \\
\hline
\end{tabular}

\section{DISCUSSION}

Bien que les cultures n'aient pas été poursuivies assez longtemps pour étudier l'effet de la salinité sur la production de grains, nous avons des indices sérieux pour prédire que le triticale « 110 » assurera une meilleure récolte que l'orge "Martin " en conditions d'excès de $\mathrm{NaCl}$ dans le sol ou l'eau d'irrigation. En effet, la nutrition du grain est assurée principalement par la photosynthèse et la remobilisation, après hydrolyse, des composés des feuilles sénescentes (Frith \& DALling, 1980); or, l'importance de ces 2 sources est fonction de la croissance foliaire, partiellement inhibée chez l'orge mais non chez le triticale.

Cette différence de sensibilité au sel ne semble pas provenir d'une meilleure régulation osmotique chez le triticale, les deux céréales résolvant de façon satisfaisante le problème posé par l'abaissement du potentiel osmotique extérieur.

Par contre, elles manifestent des comportements différents du point de vue des transports d'ions - le triticale se conduit comme une "exclusive" et l'orge comme une " inclusive" : cette dernière accumule des quantités de sodium environ 3 fois plus fortes que le
TABLEAU 3

Teneurs moyennes des parties aériennes en azote chez le triticale " 110 " et l'orge "Martin " récoltés au stade floraison, exprimées en g p. $100 \mathrm{~g} \mathrm{MS}$. Les traitements sont définis dans la légende du tableau 1. Les intervalles de confiance sont calculés pour $p=0,05$. Average nitrogen content of shoots of triticale "I10" and barley "Marn" harvested at flowering, expressed as \% DW. Treatments defined as in table 1. Confidence limits calculated for $p=0.05$.

\begin{tabular}{ccc}
\hline \hline \multirow{2}{*}{ Traitement } & \multicolumn{2}{c}{ g N p. 100 g MS } \\
\cline { 2 - 3 } & Triticale & Orge \\
\hline 0 & $0,72 \pm 0,10$ & $0,71 \pm 0,16$ \\
1,5 & $0,87 \pm 0,14$ & $0,67 \pm 0,11$ \\
3 & $0,79 \pm 0,12$ & $0,66 \pm 0,13$ \\
4,5 & $1,08 \pm 0,27$ & $0,68 \pm 0,12$ \\
$0-12$ & $0,87 \pm 0,16$ & $0,91 \pm 0,19$ \\
$1,5-12$ & $0,80 \pm 0,14$ & $0,77 \pm 0,11$ \\
$3-12$ & $0,83 \pm 0,18$ & $0,81 \pm 0,15$ \\
$4,5-12$ & $0,77 \pm 0,16$ & $0,78 \pm 0,09$ \\
\hline \hline
\end{tabular}

triticale lorsque l'on ajoute $\mathrm{NaCl}$ à la solution de culture (tabl. 1) ; le triticale conserve de ce fait un rapport $\mathrm{K} / \mathrm{Na}$ très élevé sauf dans le cas d'un traitement 
« 4,5 » ou d'un apport tardif de $\mathrm{NaCl}$ à $12 \mathrm{~g} .1^{-1}$ (fig. 3). Or l'orge est plus sensible aux traitements salés que le triticale (fig. 1). Ainsi, de ces 2 plantes, la plus résistante à $\mathrm{NaCl}$ est celle qui n'utilise pas $\mathrm{Na}^{+}$comme osmoticum, ce qui va à l'encontre du schéma habituel qui associe la tolérance au sel et l'inclusion de $\mathrm{Na}^{+}$ (MASS \& NiEMAN, 1978 ; SMITH et al., 1980 ; VAN STEVENINCK et al., 1982). L'orge rétablit son équilibre hydrique en accumulant des sels sodiques mais cette glycophyte est alors confrontée à un déséquilibre nutritionnel qui se traduit par une diminution de croissance pour chaque élévation de la concentration externe de $\mathrm{NaCl}$ (fig. 1). Par contre le triticale, grâce à sa forte sélectivité en faveur du potassium, n'encourt pas cet inconvénient ; ceci signifie que cette plante compense l'absence d'osmoticum d'origine sodique par d'autres lui permettant de résister à l'abaissement du potentiel osmotique externe. Ce comportement n'est pas totalement imprévu car le triticale « 110 » est hexaploïde, issu d'un croisement blé dur $\times$ seigle de printemps, et a donc probablement hérité du caractère de résistance à la sécheresse. L'exclusion du sodium chez le triticale s'accompagne d'une très forte sélectivité en faveur du potassium : si l'on tient compte des fréquences des apports de $\mathrm{NaCl}$ et de solution nutritive, le rapport $\mathrm{K} / \mathrm{Na}$ dans le milieu est de 0,008 pour la dose de $4,5 \mathrm{~g} \cdot 1^{-1}$ de $\mathrm{NaCl}$ et de 0,003 pour $12 \mathrm{~g} \cdot 1^{-1}$, alors qu'en moyenne, dans les organes aériens du triticale, il est 1000 fois plus élevé (fig. 3). Cette sélectivité est plus ou moins prononcée selon les organes : les teneurs en sodium des tiges et des feuilles âgées augmentent légèrement avec la concentration extérieure en $\mathrm{NaCl}$, alors que ce n'est pas le cas des dernières feuilles et que les épis accumulent des quantités croissantes de potassium (tabl. 2), ce qui se traduit par une hausse du rapport $\mathrm{K} / \mathrm{Na}$ dans ces derniers : de 32 , pour le traitement " $\mathrm{O}$ ", à 60 , pour le traitement « 4,5-12 », alors que chez l'orge, on calcule respectivement 27 et 3 (tabl. 2). Cette protection particulièrement efficace des épis du triticale s'explique peut-être par une compartimentation vacuolaire de
$\mathrm{Na}^{+}$dans les feuilles, qui fournissent une part importante de la nourriture des organes jeunes via les vaisseaux du phloème. Quelle que soit l'hypothèse retenue, les écarts entre les rapports $\mathrm{K} / \mathrm{Na}$ des feuilles âgées et ceux des organes jeunes ne peuvent pas s'expliquer par un apport de potassium empruntant seulement le xylème : en ce cas, l'apport tardif de $\mathrm{NaCl}$ à $12 \mathrm{~g} . \mathrm{l}^{-1}$ se serait traduit par une diminution de $\mathrm{K} / \mathrm{Na}$ dans les épis comme dans les feuilles; l'élucidation du mécanisme permettant au triticale de moduler le rapport $\mathrm{K} / \mathrm{Na}$ dans chaque organe passe donc par l'étude de la recirculation de l'ion $\mathrm{K}^{+}$aux stades floraison et remplissage des grains.

Les données concernant l'effet de la salinité sur le métabolisme azoté sont rares et contradictoires, mais elles s'accordent à reconnaître que le sel ne modifie pas la teneur en azote total des plantes, c'est-à-dire l'absorption de nitrate ramenée au poids de matière (Helal \& Mengel, 1979 ; LuQue \& Bingham, 1981). Nos résultats confirment ces observations, réalisées sur des plantules d'orge cultivées en hydroponique. Cette stabilité des teneurs en azote des parties aériennes de plantes telles que l'orge "Martin ", dont la masse de matière sèche diminue lorsque la dose de $\mathrm{NaCl}$ augmente, est un point remarquable : le sel affecte-t-il en tout premier lieu l'alimentation azotée qui réglerait la croissance de la plante, ou atteint-t-il surtout les synthèses glucidiques qui contrôleraient strictement l'absorption d'azote?

Notre étude débouche sur deux conclusions : la $1^{\text {re }}$, d'ordre agronomique, est que la résistance du triticale à la salinité est un argument supplémentaire pour conseiller l'extension de sa culture dans des régions à climat semi-aride comme la Tunisie; la $2^{\mathrm{e}}$, d'ordre physiologique, est que cette plante devrait constituer un matériel de choix pour les chercheurs étudiant l'effet du stress salin sur les transports ioniques.

Reçu le 14 juin 1984 Accepté le 6 décembre 1984.

\section{RÉFÉRENCES BIBLIOGRAPHIQUES}

Frith G. J. T., Dalling M. J., 1980. The role of peptide hydrolases in leaf senescence, 117-130. In K. V. Thimann : "Methods of aging research, Vol. II, Senescence in plants ». C.R.C. Press.

Greenway H., Munns R., 1980. Mechanisms of salt tolerance in nonhalophytes. Annu. Rev. Plant Physiol., 31, 149-190.

Helal H. M., Mengel K., 1979. Nitrogen metabolism of young barley plants as affected by $\mathrm{NaCl}$-salinity and potassium. Plant and Soil, 51, 457-462.

Jeschke W. D., 1979. Univalent cation selectivity and compartmentation in cereals, 37-61. In D. L. Laidman \& R. G. Wyn Jones : «Recent advances in the biochemistry of cereals ». Academic Press.

Luque A. A., Bingham F. T., 1981. The effect of the osmotic potential and specific ion concentration of the nutrient solution on the uptake and reduction of nitrate by barley seedlings. Plant and Soil, 63, 227-237.

Maas E. V., Hoffman G. J., 1977. Crop salt tolerance - Current assessment. ASCE J. Irrig. Drain. Div., 103, 115-134.

Maas E. V., Nieman R. H., 1978. Physiology of plant tolerance to salinity, 32, 277-299. In G. A. Jung : « Crop tolerance to suboptimal land conditions ".
Smith G. S., Middleton K. R., Edmonds A. S., 1980. Sodium nutrition of pasture plants. I. Translocation of sodium and potassium in relation to transpiration rates. New Phytol., 84, 603-612.

Storey R., Wyn Jones R. G., 1978. Salt stress and comparative physiology in the Gramineae. I. Ion relations of two salt - and water - stressed barley cultivars, California Mariout and Arimar. Aust. J. Plant Physiol., 5, 801-816.

Van Steveninck M. E., Stelzer R., Lauchli A., 1982. Studies on the distribution of $\mathrm{Na}$ and $\mathrm{Cl}$ in two species of lupin (Lupinus luteus and Lupinus angustifolius) differing in salt tolerance. Physiol. Plant., $56,465-473$.

Vermorel M., Bernard M., 1979. Intérêt agronomique et nutritionnel du Triticale. Bull. Techn. C.R.Z.V. Theix - I.N.R.A., 36, 31-42.

Winter E., Lauchli A., 1982. Salt tolerance of Trifolium alexandrinum L. I. Comparison of the salt response of $T$. alexandrinum and T. pratense. Aust. J. Plant Physiol., 9, 221-226.

Wyn Jones R. G., Storey R., 1978. Salt stress and comparative physiology in the Gramineae. IV. Comparison of salt stress in Spartina $X$ townsendii and three barley cultivars. Aust. J. Plant Physiol., $5,839-850$. 